\title{
Drunkorexia behaviors and motives, eating attitudes and mental health in Lebanese alcohol drinkers: a path analysis model
}

\author{
Diana Malaeb ${ }^{1}$ - Dora Bianchi ${ }^{2}$ Sara Pompili ${ }^{2}$ Jana Berro ${ }^{3}$. Fiorenzo Laghi ${ }^{2} \cdot$ Vanessa Azzi $^{4} \cdot$ Marwan Akel $^{1}$. \\ Sahar Obeid ${ }^{5} \cdot$ Souheil Hallit ${ }^{4,6}$ (B)
}

Received: 3 September 2021 / Accepted: 13 October 2021 / Published online: 29 October 2021

(c) The Author(s), under exclusive licence to Springer Nature Switzerland AG 2021

\begin{abstract}
Background Drunkorexia refers to limited intake of high-calorie food to avert gaining weight and the need to increase in alcohol excessive consumption. The present study aimed to check for an association between depression, anxiety, and stress with drunkorexia behaviors/motives among Lebanese adults, while evaluating the mediating role of inappropriate eating attitude in those associations.

Methods Two hundred and fifty-eight participants enrolled in this cross-sectional study, who filled an online survey (September-December 2020).

Results Drunkorexia behaviors were considered as the dependent variable; the path coefficients from stress to anxiety, from anxiety to eating attitudes, from eating attitudes to drunkorexia behaviors and from anxiety to drunkorexia behaviors were all significant. The indirect relationships showed that eating attitudes mediated the association between anxiety and drunkorexia behaviors. When taking the drunkorexia motives as the dependent variable, the path coefficients from stress to anxiety, from anxiety to eating attitudes, from eating attitudes to drunkorexia motives, and from anxiety to drunkorexia motives were all significant. The indirect relationships showed that eating attitudes mediated the association between anxiety and drunkorexia motives.
\end{abstract}

Conclusion This study highlighted that individuals with more psychological problems (depression, anxiety, and stress) and inappropriate eating habits exhibit more drunkorexic motivations and behaviors.

Level of evidence Level V, Cross-sectional study.

Keywords Drunkorexia $\cdot$ Disordered eating attitudes $\cdot$ Depression $\cdot$ Anxiety $\cdot$ Stress

Souheil Hallit and Sahar Obeid share the senior authorship.

Sahar Obeid

saharobeid23@hotmail.com

Souheil Hallit

souheilhallit@hotmail.com

1 School of Pharmacy, Lebanese International University, Beirut 1083, Lebanon

2 Department of Developmental and Social Psychology, Sapienza University of Rome, Rome, Italy

3 School of Medicine, Lebanese American University, Byblos, Lebanon

4 Faculty of Medicine and Medical Sciences, Holy Spirit University of Kaslik (USEK), Jounieh, Lebanon

5 Faculty of Arts and Sciences, Holy Spirit University of Kaslik (USEK), Jounieh, Lebanon

6 Research Department, Psychiatric Hospital of the Cross, Jal Eddib, Lebanon

\author{
Abbreviations \\ DMBS Drunkorexia Motives and Behaviors Scales \\ MADRS Montgomery Asberg Depression Rating Scale \\ LAS Lebanese Anxiety Scale \\ BDS Beirut Distress Scale \\ EAT Eating attitudes test
}

\section{Introduction}

Drunkorexia is the commonly used term to refer to food and alcohol disturbance [1], a high-risk behavior defined by excessive alcohol use coupled with disordered eating tendencies and/or physical activity [1]. It is characterized by the presence of one or more of the following behaviors: skipping meals or restricting food intake to "save" calories for alcohol consumption, purging behaviors such as excessive exercising as compensation for drinking, and/or consuming large 
amounts of alcohol to become ill and purge calories from alcohol consumption [2]. In other words, drunkorexia can be considered as a weight management technique to offset the consumption of calories from alcohol [3].

It is important to note that drunkorexia is a common phenomenon among the college students, where they have been reported to be around $79.1 \%$ of individuals engaging in drunkorexic behaviors [4]. Drunkorexia is triggered by different motives and characterized by various behaviors that increase alcohol consumption. Thus, the Drunkorexia Motives and Behaviors Scales is constructed to evaluate participants' engagement in drunkorexia through assessing drunkorexia motives, drunkorexia behaviors, drunkorexia fails, drunkorexia during an alcohol consumption event, and post-drinking compensation [5].

Drunkorexia behaviors are not restricted to specified time interval but can develop pre-, during, and post-alcohol consumption [6]. The potential health implications of binge drinking in drunkorexic populations are manifested by an increased alcohol toxicity, and subsequent risk of physiological damage alongside a range of psychosocial and mental health difficulties $[7,8]$.

The motivations for involvement in drunkorexia have not been thoroughly evaluated [5, 9]; however, there are four motives that explain the engagement in alcohol consumption as proposed by Cooper [10]. These drives include improvement in social interaction, development in coping strategies, enhancement, and conformity motives [11]. Drinking facilitates social interaction and relationship as it promotes a favorable social environment, and favors coping drives since it decreases negative emotions such as loneliness, boredom, and sadness. In addition, drinking exerts enhancement motives through increasing pleasure, and conformity motives through complying with social expectations.

Drunkorexia fails consist of the failure of pre-existent drunkorexic intentions, and are classified in avoidance/ approach behaviors-i.e., avoiding alcohol at all to prevent weight gain, or drinking alcohol but failing in compensative behaviors [6].

There are different important factors that are associated with both inappropriate eating and drunkorexia, including drive for thinness, body dissatisfaction, poor body esteem, ascetic tendencies, and difficulties regulating emotions [9, 12-14]. Thus, there is a close correlation between drunkorexia motive and disordered eating-referred to as "inappropriate eating" - depicted by frequent eating of palatable foods to alleviate negative affects associated with bingeeating and problematic alcohol use [15-17].

While several studies have investigated the association between psychological disorders and inappropriate eating habits in general, yet very few studies were conducted on the association between psychological distress and drunkorexia. It has been documented in previous literature that people who engage in drunkorexia showed symptoms of anxiety (Anxiety Disorders) [18] and depression (or Major Depressive Disorder) as alcohol is considered a mean of distraction from negative emotional states they are unable to bear and comfort $[19,20]$. In addition, engagement in drunkorexic behaviors may serve as a safety measure in anxious patients to decrease anxiety associated with the fear of gaining weight [21]. Not to mention, stress is highly associated with drunkorexia as stressed individuals would engage in behaviors that lower their perceived stress, including, but not limited to, restricting calories, and increasing alcohol consumption [22].

It is noteworthy to mention that higher stress commonly precedes emotional eating as individuals with higher stress tend to resort to this type of eating to escape what is worrying them [23]. Furthermore, emotional eating would be perceived by them as a rewarding feeling since it is used as a coping mechanism [24]. Anxiety and depression were also found to be associated with different eating disorders [25].

That being said, the presence of disordered eating as a common variable in drunkorexic subjects, despite other addictive behaviors [9], suggests the possibility of disordered eating playing an intermediate role between drunkorexia and factors related to psychological difficulties including depression, anxiety, and stress. This might be further explained by negative emotions of an individual about one's body weight, for example, exacerbating their partake in eating disorders, consequently participating in activities of drunkorexia.

While some countries have briefly studied the association between drunkorexia with disordered eating and problematic substance abuse [26], other countries, one of which is Lebanon-a middle-income country-have yet to approach investigating this association. Specifically, a previous study conducted in Lebanon concluded that around $28 \%$ of the adolescents are at risk of alcohol use disorder, and higher alcohol use disorder was significantly associated with higher cigarette and waterpipe smoking and bullying [27]. Furthermore, higher disordered eating attitudes and higher difficulties in emotion regulation are significantly associated with more drunkorexia motives among Lebanese adults [14]. Since Lebanese government does not have the inaction towards alcohol, in addition to the lack of existing policies regarding the illegal sale or advertisement of alcohol to minors, low excise taxes on alcohol [28], thus it is extremely vital to evaluate all factors associated with alcohol use disorder and drunkorexia among Lebanese population.

Therefore, on the basis of recent evidence $[19,20]$, the aim of this study was to determine the relation between drunkorexia and depression, anxiety, and stress in a sample of Lebanese adults, alongside with assessing the mediating role of inappropriate eating attitude $[19,20]$. According to previous studies, we specifically hypothesize 
that drunkorexia would be associated with different psychological difficulties including depression, anxiety, and stress, and that this relationship would be mediated by disordered eating attitudes. Figure 1 shows the conceptual model tested in our study.

\section{Methods}

\section{Study design and procedure}

This was a descriptive cross-sectional observational study based on an online anonymous survey. It was conducted from September until December 2020 during the second COVID-19 lockdown posed by the Lebanese government. The voluntary survey was carried out on Lebanese populations located in all Governorates of Lebanon (Beirut, Mount Lebanon, North, South, and Bekaa). To minimize interview risks and because of the lockdown restrictions enforced by the Lebanese Government, a snowball sampling method was used as an approach to the survey using online Google forms. The survey was distributed via social applications including WhatsApp, LinkedIn, and Facebook. As previously documented in the literature, research based on online questionnaire creates the opportunity to collect data on a nationwide and reach specific groups of individuals [29, 30]. All the invited participants were

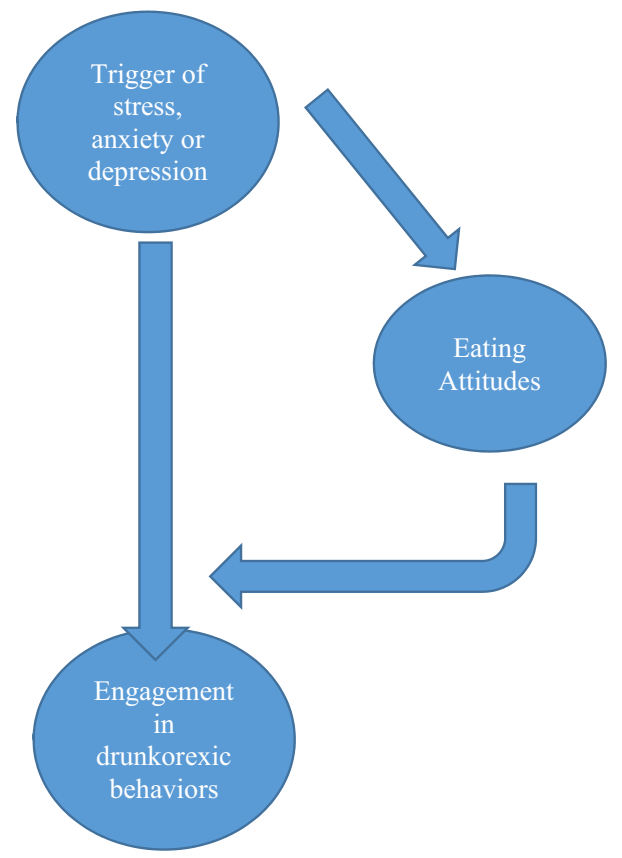

Fig. 1 Conceptual model explaining the engagement in drunkorexic behaviors above 18 years of age. The same methodology is described elsewhere [14].

\section{Minimal sample size calculation}

Based on a $79.1 \%$ of patients reported engaging in drunkorexia [4] and in the absence of similar studies in Lebanon, the minimal sample size calculated according to the Epi Info software version 7.2 (population survey) was 254 participants to ensure a confidence level of $95 \%$.

\section{Questionnaire}

The self-administered questionnaire used was in Arabic, Lebanon's official and native language, and required approximately $20 \mathrm{~min}$ to be completed. Participants were asked to fill out the questionnaire without the request of any help to avoid any potential influence when answering the questions. The anonymity of the participants was guaranteed during the data collection process.

The first part of the questionnaire evaluated participants' sociodemographic information (age, marital status and educational level). Educational level was categorized into complementary, secondary, and university level.

The second part of the questionnaire composed of the different scales used:

\section{Drunkorexia Motives and Behaviors Scales (DMBS)}

The DMBS contains a total of 52 items that evaluate participants' engagement in drunkorexia [5]. Each item includes the prompt "Rate the frequency of each statement" and the items are on a Likert type-scale including never (1), seldom (2), sometimes (3), often (4), and very often (5). In the present study, only two dimensions from the DMBS have been used: drunkorexia motives (15 items), classified into the reasons why individuals engage in drunkorexia (e.g., "Because it helps me enjoy a party"), and drunkorexia behaviors (8 items) that relate to different behaviors associated with drunkorexia (e.g., "By exercising more than normal"). Both dimensions reached excellent values of reliability in this study (Drunkorexia motives Cronbach's $\alpha=0.957$, Drunkorexia behaviors Cronbach's $\alpha=0.950$ ).

\section{Montgomery Asberg Depression Rating Scale (MADRS)}

MADRS is a 10-item semi-structured validated scale among Lebanese population and serve to indicate the severity of the depressive condition. The MADRS assesses mood symptoms exhibited over the preceding 2 weeks, scoring items from 0 to 6 , to give a maximum total score of 60 points. Müller proposed the following cutoff points for severity: $0-8=$ remission $; 9-17=$ mild; $18-34=$ moderate; and more 
than $35=$ severe [31]. The items assess somatic, cognitive, vegetative and anxious symptoms. Higher scores indicate increasing depressive symptoms. This scale is validated in Lebanon [32]. In the present study, the Cronbach's $\alpha$ values was 0.865 .

\section{Lebanese Anxiety Scale (LAS)}

The Lebanese Anxiety Scale, inspired by the diagnostic criteria of DSM-5, and the HAM-A and STAI scales [18, 33], consists of 10 items, with 7 questions rated from 0 (symptoms not present) to 4 (very severe symptoms) and three questions from 0 to 3 ; higher scores reflect higher anxiety [18]. In this study, the Cronbach's alpha was 0.915.

\section{Beirut Distress Scale (BDS)}

BDS-10 is a validated scale, used to assess psychological distress [34]. Answers were recorded on a 4-point Likert scale from 0 (never) to 3 (very much), with higher scores indicating higher stress. In this study, the Cronbach's alpha was 0.877 .

\section{Eating attitudes test (EAT)}

The EAT, recently validated in Lebanon [35], is a 26-item questionnaire that is used to measure abnormal eating behaviors and concerns about weight. The participants rated their feelings towards eating behavior and weight concerns. The EAT is scored for a total of all of the items (e.g., "I am preoccupied with a desire to be thinner," "I feel extremely guilty after eating," and "I have the impulse to vomit after meals"). In addition, the EAT has three subscales: Dieting, Bulimia and Food Preoccupation, and Oral Control. Participants responded to the items using a 6-point Likert scale with choices including: "Never," "Rarely," "Sometimes," "Often," "Very Often," and "Always." The choices of "Never," "Rarely," and "Sometimes" were scored as zero and the rest of the choices were scored 1,2, and 3, respectively. A score above 20 is viewed as a sign of an eating disorder problem. In the current sample, the Cronbach's $\alpha$ for the total scale was 0.969 [36].

\section{Translation procedure}

A clinical psychologist performed the forward translation from English into Arabic for the DMBS. A professional medical writer verified this translation. The backward translation was performed by a second clinical psychologist, unaware of the scales' notions and fluent in Arabic. The principal investigator matched the back-translated English questionnaire with the original one to detect inconsistencies and solve discrepancies between the two versions.

\section{Statistical analysis}

All analyses were carried out using the Statistical Package for Social Sciences (version 24.0 with AMOS; IBM ${ }^{\circledR}$, Armonk, NY, USA). Path analysis modeling was performed to examine the structural relationship between mental health issues, eating attitudes and drunkorexia motives/behaviors. First, the assessment of normal distribution of the drunkorexia motives/behaviors scores was performed; this was verified by calculation of the skewness and kurtosis; values for asymmetry and kurtosis between -1 and +1 are considered acceptable to prove normal univariate distribution [37]. These conditions consolidate the assumptions of normality in samples larger than 300 [38]. Several scores were calculated to assess the goodness-of-fit of the model: the root mean square error of approximation (RMSEA) statistic, Tucker Lewis Index (TFI) and the comparative fit index (CFI) were used to evaluate the goodness-of-fit of the model [32]. RMSEA values $\leq 0.06$ or CFI and TFI values $>0.90$ indicate a good-fitting model [32]. $p<0.05$ was considered statically significant.

\section{Results}

Two hundred and fifty-eight participants out of 340 (75.88\%) completed the survey (age 26.96 \pm 9.39 years; range 18-80 years, and $21.3 \%$ females). Other details about the sample can be found in Table 1 . The general model that was tested is summarized in Fig. 2.

\section{Drunkorexia behaviors}

The fit indices of the three tested models for the drunkorexia behaviors are presented in Table 2. As can be seen, model 1 that included stress and anxiety only fitted well according to RMSEA, CFI, TLI and $\chi^{2} / d f$ values, whereas model 2 (taking stress and depression) and model 3 (taking stress, anxiety and depression) fitted poorly.

\section{Path analysis modeling}

The relationships between stress, anxiety, eating attitudes and drunkorexia behaviors are shown in Fig. 3. The path coefficients from stress to anxiety, from anxiety to eating attitudes, from eating attitudes to drunkorexia behaviors 
Table 1 Sociodemographic and other characteristics of the participants $(N=258)$

\begin{tabular}{ll}
\hline Variable & $\mathrm{N}(\%)$ \\
\hline Gender & $203(78.7 \%)$ \\
Male & $55(21.3 \%)$ \\
Female & \\
Marital status & $203(78.7 \%)$ \\
Single & $55(21.3 \%)$ \\
Married & $32(12.4 \%)$ \\
Education level & $34(13.2 \%)$ \\
Complementary or less & $192(74.4 \%)$ \\
Secondary & Mean \pm SD \\
University & $26.96 \pm 9.39$ \\
\hline & $18.88 \pm 9.28$ \\
Age (in years) & $12.77 \pm 7.25$ \\
Anxiety & $14.45 \pm 12.64$ \\
Stress & $31.54 \pm 27.78$ \\
Depression & $20.88 \pm 14.46$ \\
EAT-26 & $13.28 \pm 8.64$ \\
Drunkorexia motives & \\
Drunkorexia behaviors & \\
\hline
\end{tabular}

$E A T$ eating attitude test, $S D$ standard deviation

and from anxiety to drunkorexia behaviors were all significant. The indirect relationships showed that eating attitudes mediated the association between anxiety and drunkorexia behaviors (Table 3).

\section{Drunkorexia motives}

The fit indices of the three tested path analysis models for the drunkorexia motives are presented in Table 4. As can be seen, model 1 that included stress and anxiety only fitted well according to RMSEA, CFI, TLI and $\chi^{2} / d f$ values, whereas model 2 (taking stress and depression) and model 3 (taking stress, anxiety and depression) fitted poorly.

\section{Path analysis modeling}

The relationships between stress, anxiety, eating attitudes and drunkorexia behaviors are shown in Fig. 4. The path coefficients from stress to anxiety, from anxiety to eating attitudes, from eating attitudes to drunkorexia motives and from anxiety to drunkorexia motives were all significant. The indirect relationships showed that eating attitudes mediated the association between anxiety and drunkorexia motives (Table 5).

\section{Discussion}

With very few studies investigating drunkorexia, a rising phenomenon among adults, our study focused on the association between drunkorexia and psychological disorders while emphasizing on the role of inappropriate eating in mediating this relationship. The importance of investigating this type of behavior lies within the consequences it poses on one's physical and mental health [39]. The detrimental effects of alcohol alone include dehydration, malnutrition, depression and anxiety. When paired with inappropriate eating habits, these effects are exaggerated to include cardiovascular problems in addition to suicidal thoughts [40].

Our study found that individuals with inappropriate eating behaviors report higher drunkorexia motives and behaviors. A previous study by Barry and Piazza-Gardner [41] has also found that disordered eating was statistically significantly associated with drinking. This could happen since individuals who partake in inappropriate eating habits usually do not differentiate between feeling of hunger, satiety, and other visceral sensations [42]. Drunkorexia is considered to include the overlap of inappropriate eating habits and alcohol use [43, 44]. Those who reduced calorie consumption prior to alcohol have also scored higher on the disordered eating score [19]. Studies have also found that both disordered eating and alcohol use strongly predict an individual's engagement in drunkorexic behaviors [45]. While it has been shown in several studies that this 


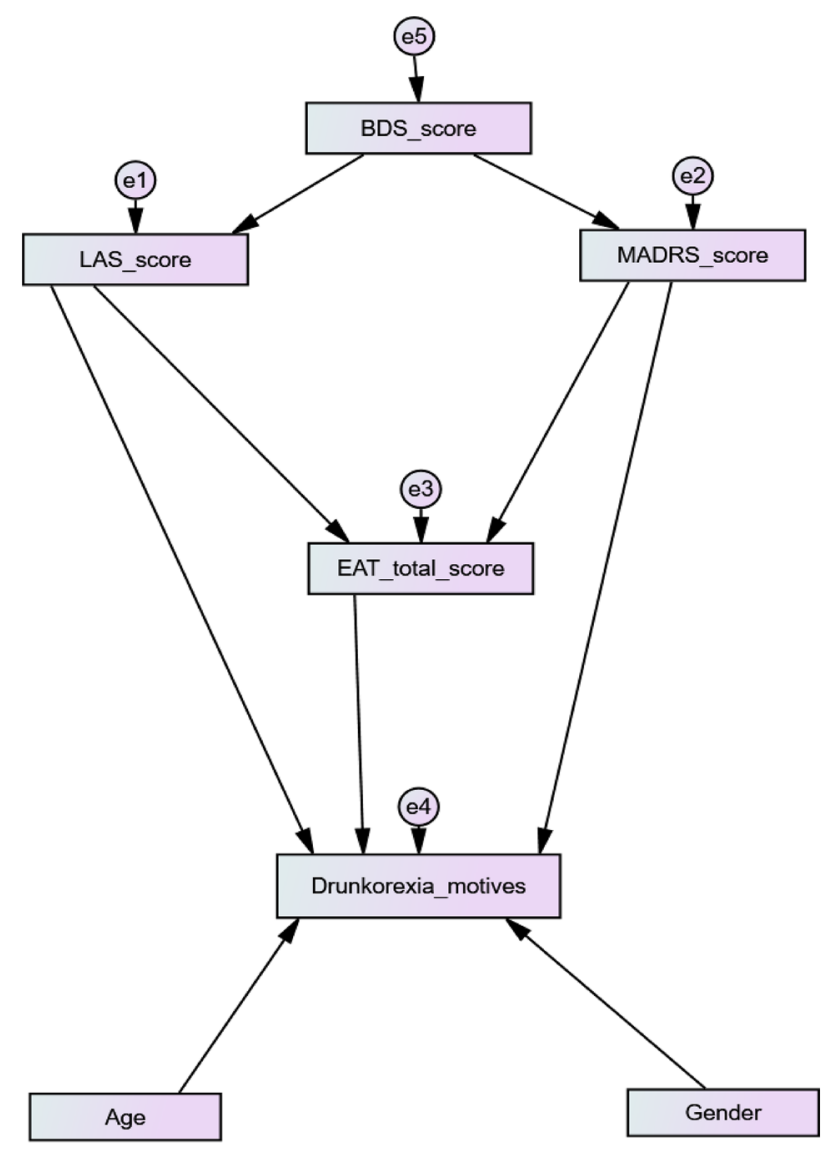

Fig. 2 Path analysis model in Lebanese adults. $\square$-observed variable; $\bigcirc$-latent variable; $\longrightarrow$-impact of one variable on another; e-residual error in the prediction of an unobserved factor. EAT eating attitudes, MADRS depression score, LAS anxiety score, $B D S$ stress score

correlation is stronger among females than males [9], our results did not find a significant role played by the sex of the individual. It is noteworthy to note that $78.7 \%$ of our participants were males, which may have contributed to this discrepancy.

Our study also found that individuals with high stress and anxiety were associated with drunkorexic motives and behaviors. This is in accordance with other findings that have shown these psychological states' triggering effect on alcohol consumption and consequential drunkorexia [46].
Several other studies have already investigated and established how drinking is considered one of the maladaptive behaviors implemented by adults dealing with difficulties regulating their intense emotions [47].

A previous study has shown that depression has been correlated to drunkorexia motives and behaviors [19]. Several theories are postulated as to the reason behind this, mainly focusing on how people with depression tend to get involved in eating behavior disorders as means of distraction from thoughts related to negative self-perception and self-criticism regarding weight and body shape [48]. However, our results suggested that depression was not implied in the psychological disorders triggering drunkorexia via dysfunctional eating attitudes. This evidence should be further explored in future studies to understand the possible role of other interacting variables.

On the other hand, disordered eating partially mediated the association between anxiety and drunkorexia motives and behaviors. As suggested in previous research, individuals tend to restrict caloric consumption because of their fear of gaining weight [21]. Another study showed that without adequate and proper coping mechanisms, individuals with anxiety are more likely to participate in drunkorexic behaviors [20]. Furthermore, a study showed that patients who exhibit calorie restriction and purging behaviors have some dysfunction within their hypothalamic-pituitary-adrenal (HPA) axis [49]. Another study has also found that stressful emotions being a potential predictor of drunkorexic behavior or motives could be explained by individuals resorting to this behavior when lack the necessary skills of dealing with different types of strong emotions as it makes them feel in control [13].

A previous study has discussed how negative self-perception and an individual's unacceptance of his body image could play a role in developing drunkorexia, as a way of coping with his depression [50]. This might also be used to hypothesize that if individuals experiencing depressive emotions also experience drunkorexia fails, they might resort to even further caloric restrictions as compensatory behaviors. Furthermore, Eisenberg and Fitz [51] found that this relationship between drunkorexia and disordered eating can be explained by their extreme desire to achieve a weight and body shape positively accepted by society in addition to emotions of fear of weight gain. In addition, it is well known
Table 2 Fit indices of the path analysis for different models related to drunkorexia behaviors

\begin{tabular}{lllllllr}
\hline & $\chi_{(d f)}^{2}$ & $p$ & TLI & CFI & RMSEA & $90 \%$ CI & \multicolumn{1}{c}{$p_{\text {close }}$} \\
\hline Model 1 & $41.71_{(9)}$ & $<0.001$ & 0.932 & 0.971 & 0.05 & $0.04,0.07$ & 0.266 \\
Model 2 & $52.69_{(9)}$ & $<0.001$ & 0.621 & 0.838 & 0.06 & $0.05,0.08$ & 0.071 \\
Model 3 & $144.30_{(12)}$ & $<0.001$ & 0.782 & 0.907 & 0.10 & $0.08,0.11$ & $<0.001$ \\
\hline
\end{tabular}

Model $1=$ stress and anxiety alone; model $2=$ stress and depression alone; model $3=$ stress, anxiety and depression 


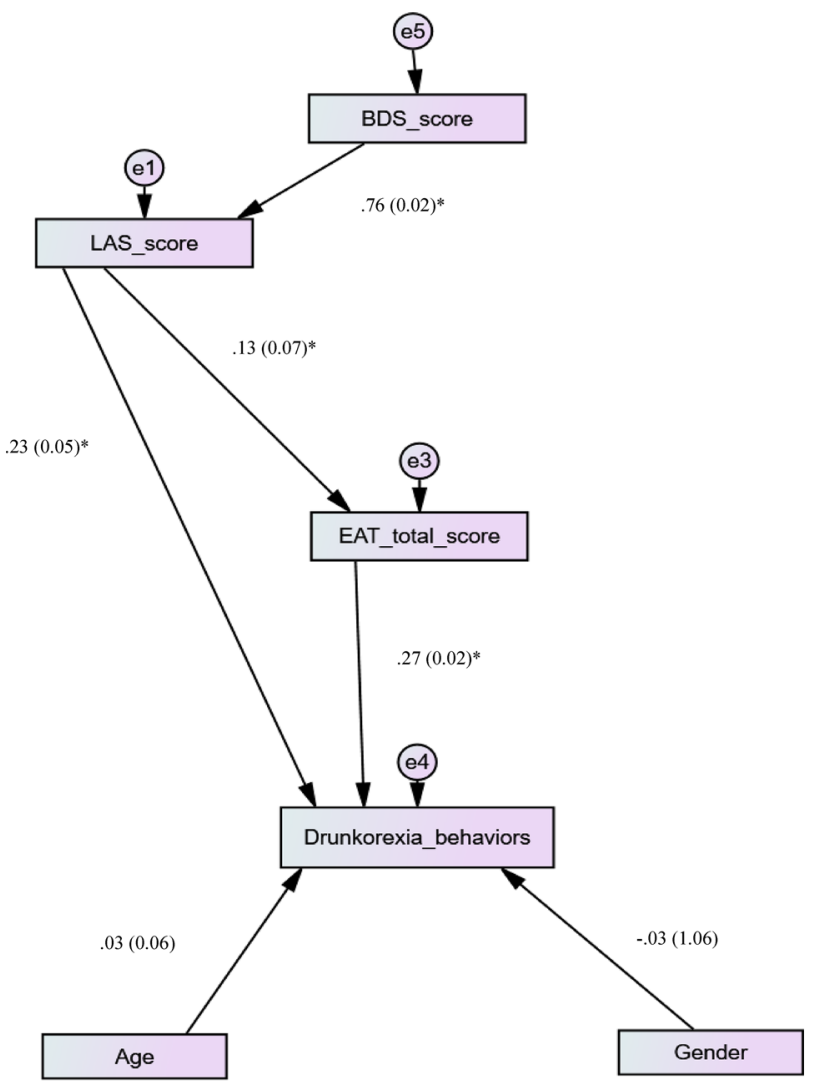

Fig. 3 Path analysis model in Lebanese adults related to drunkorexia behaviors. - observed variable; $\bigcirc$-latent variable; $\longrightarrow$-impact of one variable on another; e- residual error in the prediction of an unobserved factor. ${ }^{*} p<0.001$. EAT eating attitudes, $L A S$ anxiety score, $B D S$ stress score that disordered eating behaviors, such as self-induced vomiting and other calorie-restricting behaviors, have been used by adults with emotion-regulating difficulties [52].

\section{What is already known about the topic?}

Drunkorexia is an increasing phenomenon that has not been studied extensively and fully understood. There have been several theories relating the motives behind drunkorexia to individuals desiring conformity and/or improving of social status. It has also been theorized to be a coping mechanism in one's need to enhance positive affect. Based on this, several studies have associated drunkorexia with psychological disorders in general, especially those in which one cannot properly regulate his/her own intense emotions.

\section{What does this study add?}

Our study dove deeper into the association between different psychological states and drunkorexia. Stress and anxiety were associated with more drunkorexic motives and behaviors, as were inappropriate eating habits. The latter could be acting as a mediating factor between the former two as we hypothesize the reason behind that is that individuals who are anxious about and fear gaining weight would resort to inappropriate eating habits, eventually restricting caloric intake before consuming alcohol. Depression has been associated with drunkorexia fails; it is hypothesized that of the compensatory mechanisms is even further caloric restriction.
Table 3 Coefficient, standard error and $p$ value of the path analysis model taking the drunkorexia behaviors as the dependent variable
Table 4 Fit indices of the path analyses for different models related to drunkorexia motives

\begin{tabular}{lcrr}
\hline Variable & $\begin{array}{l}\text { Standardized regression } \\
\text { coefficient }\end{array}$ & Standard error & $p$ \\
\hline Stress $\rightarrow$ anxiety & 0.76 & 0.02 & $\mathbf{< 0 . 0 0 1}$ \\
Anxiety $\rightarrow$ eating attitudes & 0.13 & 0.07 & $\mathbf{< 0 . 0 0 1}$ \\
Anxiety $\rightarrow$ drunkorexia behaviors & 0.23 & 0.05 & $\mathbf{< 0 . 0 0 1}$ \\
Eating attitudes $\rightarrow$ drunkorexia behaviors & 0.27 & 0.02 & $\mathbf{< 0 . 0 0 1}$ \\
Age $\rightarrow$ drunkorexia behaviors & 0.03 & 0.06 & 0.623 \\
Gender $\rightarrow$ drunkorexia behaviors & -0.03 & 1.06 & 0.661 \\
\hline
\end{tabular}

Numbers in bold indicate significant $p$ values

\begin{tabular}{lllllllc}
\hline & $\chi^{2}(d f)$ & $p$ & TLI & CFI & RMSEA & $90 \%$ CI & $p_{\text {close }}$ \\
\hline Model 1 & $41.40_{(9)}$ & $<0.001$ & 0.934 & 0.972 & 0.05 & $0.04,0.07$ & 0.275 \\
Model 2 & $58.60_{(9)}$ & $<0.001$ & 0.610 & 0.833 & 0.07 & $0.05,0.09$ & 0.03 \\
Model 3 & $144.13_{(12)}$ & $<0.001$ & 0.786 & 0.908 & 0.10 & $0.08,0.11$ & $<0.001$ \\
\hline
\end{tabular}

Model $1=$ stress and anxiety alone; model $2=$ stress and depression alone; model $3=$ stress, anxiety and depression 


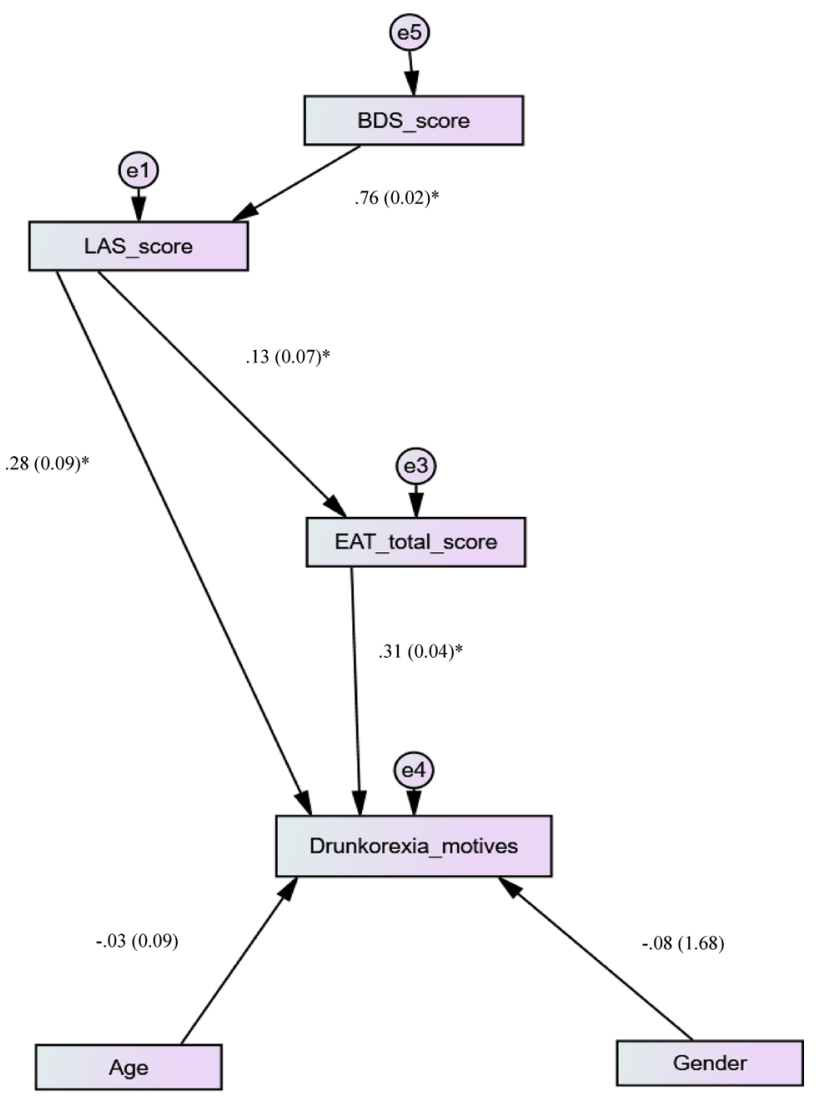

Fig. 4 Path analysis model in Lebanese adults related to drunkorexia motives. $\longrightarrow$-observed variable; $\bigcirc$-latent variable; $\longrightarrow$ -impact of one variable on another; e-residual error in the prediction of an unobserved factor. ${ }^{*} p<0.001$. EAT eating attitudes, $L A S$ anxiety score, $B D S$ stress score

\section{Study implications and further research}

The results of this study might serve as a first step towards organizing awareness campaigns about drinking behaviors for highly vulnerable groups aiming at reducing the prevalence of drinking patterns. This study also shed light on the relevance of implementation of integrated programs to minimize drinking habit and reinforce healthy lifestyle practices. Interventional and educational programs should motivate clinicians, parents and friends to encourage individuals to abstain from engagement in such behavior. Furthermore, this study opened doors for future research to depict a clear association between disordered eating, mental health and drunkorexia and evaluate its long-term effects.

\section{Limitations and strengths}

Due to the cross-sectional design of our study, we were unable to explore causal associations; thus, results are needed to be examined with the implementation of a longitudinal design to test the course and long-term outcome of drunkorexia. In addition, the study was performed during COVID19 lockdown and confinement that can influence our study results: on one hand, the lockdown condition may have exacerbated psychological stress and anxiety, leading to more problematic eating and drinking behaviors. On the other hand, many people were home confined with their families, and recent research has shown that living with family during the pandemic was a protective factor, reducing the engagement in alcohol consumption [51]. This research relied on self-report measures, thus, data may be subjected to inaccuracy and potential bias in the responses. To note also is the higher number of males compared to females in our study that may not reflect the gender component of drunkorexia effects. The results obtained suggest that these patterns could be adequate for males only, while different models might emerge in a broader sample of females. A selection bias is possible because of the convenient sampling technique used to recruit participants. A residual confounding bias is also possible since not all factors associated with drunkorexia were considered in this paper; the COVID-19 pandemic and its consequent lockdowns were associated with higher mental health issues (depression, anxiety, stress, obsession, etc.) among the Lebanese population [53]. The economic crisis, lack of clean water, recurrent power failure, and waste mismanagement and Syrian refugees immigration to Lebanon $[54,55]$ have also impacted psychological behaviors, possibly affecting our findings. However, this study has several important strengths. First, to our knowledge, the research on
Table 5 Coefficient, standard error and $p$ value of the path analysis model taking the drunkorexia motives as the dependent variable

\begin{tabular}{lccr}
\hline Variable & $\begin{array}{l}\text { Standardized regression } \\
\text { coefficient }\end{array}$ & Standard error & $p$ \\
\hline Stress $\rightarrow$ anxiety & 0.76 & 0.02 & $\mathbf{< 0 . 0 0 1}$ \\
Anxiety $\rightarrow$ eating attitudes & 0.13 & 0.07 & $\mathbf{< . 0 0 1}$ \\
Anxiety $\rightarrow$ drunkorexia motives & 0.28 & 0.09 & $\mathbf{< 0 . 0 0 1}$ \\
Eating attitudes $\rightarrow$ drunkorexia motives & 0.31 & 0.04 & $\mathbf{< 0 . 0 0 1}$ \\
Age $\rightarrow$ drunkorexia motives & -0.03 & 0.09 & 0.570 \\
Gender $\rightarrow$ drunkorexia motives & -0.08 & 1.68 & 0.167 \\
\hline
\end{tabular}

Numbers in bold indicate significant $p$ values 
drunkorexia is very limited in our region and this research project is a pioneer in the field. Furthermore, this study was the first to analyze the relation between drunkorexia, depression, anxiety and stress and disordered eating behaviors and explore the association of drunkorexia with dimensions of mental health.

\section{Conclusion}

Drunkorexia is a rising trend among adolescents and adults; however, it is seldom investigated, especially in relation to psychological distress as a triggering factor rather than a consequence. Our study investigated the relationship between depression, anxiety or stress and drunkorexia, while focusing the role disordered eating might have on this association, among the Lebanese population. This relationship is imperative to be well studied as the consequences of drunkorexia, especially with co-current disordered eating can have life-threatening consequences on an individual. Our results found that individuals dealing with intense emotions, such as the aforementioned, tend to exhibit higher drunkorexic motivations and behaviors. Higher scores on inappropriate eating habits scales were also correlated with higher drunkorexic behaviors. These results along with previous literature could explain the possibility of individuals who are experience peer pressure and dissatisfaction with their body image engaging in disordered eating behaviors and drunkorexia as means of coping with their emotions. It is important that this association is further investigated to plan accordingly as these dysfunctional habits can have life-threatening consequences.

Acknowledgements The authors would like to thank all the participants.

Author contributions $\mathrm{SO}$ and $\mathrm{SH}$ conceived and designed the survey. $\mathrm{SH}$ was involved in the statistical analysis and data interpretation. DM and JB wrote the manuscript. MA was involved in the data collection; MA, VA, DB, SP and FL reviewed the manuscript. All the authors read the manuscript, critically revised it for intellectual content, and approved the final version.

Funding None.

Availability of data and materials The authors do not have the right to share any data information as per their institutions' policies.

\section{Declarations}

Conflict of interest The authors have no conflicts of interest to report.

Ethics approval The Psychiatric Hospital of the Cross ethics committee approved the study protocol (HPC-042-2020).
Consent to participate Submitting the form online was equivalent to obtaining a written informed consent from each participant.

Consent for publication Not applicable.

\section{References}

1. Piazza-Gardner AK, Barry AE (2013) Appropriate terminology for the alcohol, eating, and physical activity relationship. J Am Coll Health 61:311-313

2. Barry AE, Whiteman S, Piazza-Gardner AK, Jensen AC (2013) Gender differences in the associations among body mass index, weight loss, exercise, and drinking among college students. J Am Coll Health 61:407-413

3. Thompson-Memmer C, Glassman T, Diehr A (2019) Drunkorexia: a new term and diagnostic criteria. J Am Coll Health 67:620-626

4. Knight A, Simpson S (2013) Drunkorexia: an empirical investigation of disordered eating in direct response to saving calories for alcohol use amongst Australian female university students. J Eat Disord 1:1-1

5. Ward RM, Galante M (2015) Development and initial validation of the Drunkorexia Motives and Behaviors scales. Eat Behav 18:66-70

6. Knight A, Castelnuovo G, Pietrabissa G, Manzoni GM, Simpson S (2017) Drunkorexia: an empirical investigation among Australian female university students. Aust Psychol 52:414-423

7. Ham LS, Hope DA (2003) College students and problematic drinking: a review of the literature. Clin Psychol Rev 23:719-759

8. Burke LM, Collier GR, Broad EM, Davis PG, Martin DT, Sanigorski AJ, Hargreaves M (2003) Effect of alcohol intake on muscle glycogen storage after prolonged exercise. J Appl Physiol (1985) 95:983-990. https://doi.org/10.1152/japplphysiol.00115.2003

9. Pompili S, Laghi F (2018) Drunkorexia among adolescents: the role of motivations and emotion regulation. Eat Behav 29:1-7

10. Cooper ML (1994) Motivations for alcohol use among adolescents: development and validation of a four-factor model. Psychol Assess 6:117

11. Gmel G, Labhart F, Fallu JS, Kuntsche E (2012) The association between drinking motives and alcohol-related consequences-room for biases and measurement issues? Addiction 107:1580-1589

12. Hill EM, Lego JE (2020) Examining the role of body esteem and sensation seeking in drunkorexia behaviors. Eat Weight Disord 25:1507-1513

13. Laghi F, Pompili S, Bianchi D, Lonigro A, Baiocco R (2020) Psychological characteristics and eating attitudes in adolescents with drunkorexia behavior: an exploratory study. Eat Weight Disord 25:709-718

14. Azzi V, Hallit S, Malaeb D, Obeid S, Brytek-Matera A (2021) Drunkorexia and emotion regulation and emotion regulation difficulties: the mediating effect of disordered eating attitudes. Int J Environ Res Public Health 18(5):2690. https://doi.org/10.3390/ ijerph 18052690

15. Fazzino TL, Raheel A, Peppercorn N, Forbush K, Kirby T, Sher KJ, Befort C (2018) Motives for drinking alcohol and eating palatable foods: an evaluation of shared mechanisms and associations with drinking and binge eating. Addict Behav 85:113-119

16. Jackson KM, Sher KJ (2003) Alcohol use disorders and psychological distress: a prospective state-trait analysis. J Abnorm Psychol 112:599-613

17. Simons JS, Simons RM, O'Brien C, Stoltenberg SF, Keith JA, Hudson JA (2017) PTSD, alcohol dependence, and conduct 
problems: distinct pathways via lability and disinhibition. Addict Behav 64:185-193

18. Hallit S, Obeid S, Haddad C, Hallit R, Akel M, Haddad G, Soufia M, Khansa W, Khoury R, Kheir N, Abi Elias Hallit C, Sacre H, Salameh P (2020) Construction of the Lebanese Anxiety Scale (LAS-10): a new scale to assess anxiety in adult patients. Int J Psychiatry Clin Pract 24:270-277

19. Roosen KM, Mills JS (2015) Exploring the motives and mental health correlates of intentional food restriction prior to alcohol use in university students. J Health Psychol 20:875-886

20. Laghi F, Pompili S, Bianchi D, Lonigro A, Baiocco R (2021) Exploring the association between psychological distress and drunkorexia behaviors in non-clinical adolescents: the moderating role of emotional dysregulation. Eat Weight Disord 26:797-806

21. Pallister E, Waller G (2008) Anxiety in the eating disorders: understanding the overlap. Clin Psychol Rev 28:366-386

22. Oswald BB, Ward RM, Glazer S, Sternasty K, Day K, Speed S (2020) Baseline cortisol predicts drunkorexia in female but not male college students. J Am Coll Health 69(6):625-632. https:// doi.org/10.1080/07448481.2019.1705834

23. Van Blyderveen S, Lafrance A, Emond M, Kosmerly S, O'Connor M, Chang F (2016) Personality differences in the susceptibility to stress-eating: the influence of emotional control and impulsivity. Eat Behav 23:76-81

24. Royal JD, Kurtz JL (2010) I ate what?! The effect of stress and dispositional eating style on food intake and behavioral awareness. Personal Individ Differ 49:565-569

25. Melchior C, Desprez C, Riachi G, Leroi AM, Dechelotte P, Achamrah N, Ducrotte P, Tavolacci MP, Gourcerol G (2019) Anxiety and depression profile is associated with eating disorders in patients with irritable bowel syndrome. Front Psychiatry 10:928

26. Simons RM, Hansen JM, Simons JS, Hovrud L, Hahn AM (2021) Drunkorexia: normative behavior or gateway to alcohol and eating pathology? Addict Behav 112:106577

27. Hallit J, Salameh P, Haddad C, Sacre H, Soufia M, Akel M, Obeid S, Hallit R, Hallit S (2020) Validation of the AUDIT scale and factors associated with alcohol use disorder in adolescents: results of a National Lebanese Study. BMC Pediatr 20:205

28. Yassin N, Afifi R, Singh N, Saad R, Ghandour L (2018) “There is zero regulation on the selling of alcohol": the voice of the youth on the context and determinants of alcohol drinking in Lebanon. Qual Health Res 28:733-744

29. Wright KB (2005) Researching internet-based populations: advantages and disadvantages of online survey research, online questionnaire authoring software packages, and web survey services. J Comput Mediat Commun 10:00-00

30. Rzymski P, Jaśkiewicz M (2017) Microalgal food supplements from the perspective of Polish consumers: patterns of use, adverse events, and beneficial effects. J Appl Phycol 29:1841-1850

31. Muller MJ, Himmerich H, Kienzle B, Szegedi A (2003) Differentiating moderate and severe depression using the Montgomery-Asberg depression rating scale (MADRS). J Affect Disord 77:255-260

32. Hallit S, Obeid S, El Hage W, Kazour F (2019) Validation of the Arabic version of the MADRS scale among Lebanese patients with depression. Encephale 45:195-199

33. Merhy G, Azzi V, Salameh P, Obeid S, Hallit S (2021) Anxiety among Lebanese adolescents: scale validation and correlates. BMC Pediatr 21(1):288. https://doi.org/10.1186/ s12887-021-02763-4

34. Malaeb D, Farchakh Y, Haddad C, Sacre H, Obeid S, Hallit S, Salameh P (2021) Validation of the Beirut Distress Scale (BDS10 ), a short version of BDS-22, to assess psychological distress among the Lebanese population. Perspect Psychiatr Care. https:// doi.org/10.1111/ppc.12787
35. Haddad C, Khoury C, Salameh P, Sacre H, Hallit R, Kheir N, Obeid S, Hallit S (2020) Validation of the Arabic version of the Eating Attitude Test in Lebanon: a population study. Public Health Nutr 24(13):4132-4143. https://doi.org/10.1017/S136898002 0002955

36. Garner DM, Olmsted MP, Bohr Y, Garfinkel PE (1982) The eating attitudes test: psychometric features and clinical correlates. Psychol Med 12:871-878

37. Hair JF Jr, Hult GTM, Ringle C, Sarstedt M (2017) A primer on partial least squares structural equation modeling (PLS-SEM). Sage Publications, New York

38. Mishra P, Pandey CM, Singh U, Gupta A, Sahu C, Keshri A (2019) Descriptive statistics and normality tests for statistical data. Ann Card Anaesth 22:67-72

39. Powell-Jones A, Simpson S (2020) Drunkorexia: an investigation of symptomatology and early maladaptive schemas within a female, young adult Australian population. Aust Psychol 55:559-571

40. Lupi M, Acciavatti T, Santacroce R, Cinosi E, Martinotti G, Di Giannantonio M (2014) "Drunkorexia": a pilot study in an Italian sample. Res Adv Psychiatry 1:1-5

41. Barry AE, Piazza-Gardner AK (2012) Drunkorexia: understanding the co-occurrence of alcohol consumption and eating/exercise weight management behaviors. J Am Coll Health 60:236-243

42. Preyde M, Watson J, Remers S, Stuart R (2016) Emotional dysregulation, interoceptive deficits, and treatment outcomes in patients with eating disorders. Soc Work Ment Health 14:227-244

43. Laghi F, Baiocco R, Liga F, Lonigro A, Baumgartner E (2014) Binge eating and binge drinking behaviors: individual differences in adolescents' identity styles. J Health Psychol 19:333-343

44. Laghi F, McPhie ML, Baumgartner E, Rawana JS, Pompili S, Baiocco R (2016) Family functioning and dysfunctional eating among Italian adolescents: the moderating role of gender. Child Psychiatry Hum Dev 47:43-52

45. Hunt TK, Forbush KT (2016) Is "drunkorexia" an eating disorder, substance use disorder, or both? Eat Behav 22:40-45

46. Foster S, O'Mealey M, Farmer C, Carvallo M (2020) The impact of snapchat usage on drunkorexia behaviors in college women. J Am Coll Health 1:1-11. https://doi.org/10.1080/07448481.2020. 1775609

47. Boggiano MM, Burgess EE, Turan B, Soleymani T, Daniel S, Vinson LD, Lokken KL, Wingo BC, Morse A (2014) Motives for eating tasty foods associated with binge-eating. Results from a student and a weight-loss seeking population. Appetite 83:160-166

48. Dunkley DM, Grilo CM (2007) Self-criticism, low self-esteem, depressive symptoms, and over-evaluation of shape and weight in binge eating disorder patients. Behav Res Ther 45:139-149

49. Ginty AT, Phillips AC, Higgs S, Heaney JL, Carroll D (2012) Disordered eating behaviour is associated with blunted cortisol and cardiovascular reactions to acute psychological stress. Psychoneuroendocrinology 37:715-724

50. Osborne V, Sher K, Winograd R (2011) Disordered eating patterns and alcohol misuse in college students: evidence for "drunkorexia"? Compr Psychiatry 6:e12

51. Eisenberg MH, Fitz CC (2014) "Drunkorexia": exploring the who and why of a disturbing trend in college students' eating and drinking behaviors. J Am Coll Health 62:570-577

52. Haynos AF, Wang SB, Fruzzetti AE (2018) Restrictive eating is associated with emotion regulation difficulties in a non-clinical sample. Eat Disord 26:5-12

53. El Othman R, Touma E, El Othman R, Haddad C, Hallit R, Obeid S, Salameh P, Hallit S (2021) COVID-19 pandemic and mental health in Lebanon: a cross-sectional study. Int J Psychiatry Clin Pract 25:152-163 
54. Cherri Z, Arcos GP, Castro DR (2016) The Lebanese-Syrian crisis: impact of influx of Syrian refugees to an already weak state. Risk Manag Healthc Policy 9:165-172

55. Obeid S, Haddad C, Salame W, Kheir N, Hallit S (2019) Xenophobic attitudes, behaviors and coping strategies among Lebanese people toward immigrants and refugees. Perspect Psychiatr Care 55:710-717
Publisher's Note Springer Nature remains neutral with regard to jurisdictional claims in published maps and institutional affiliations. 